\title{
Normal mediastinal and hilar lymph nodes in children on multi-detector row chest computed tomography
}

\author{
Pim A. de Jong $\cdot$ Rutger-Jan A. Nievelstein
}

Received: 29 June 2011 /Revised: 8 August 2011 / Accepted: 11 August 2011 / Published online: 2 September 2011

(C) The Author(s) 2011. This article is published with open access at Springerlink.com

\begin{abstract}
Objective To study normal mediastinal and hilar lymph nodes on multi-detector chest computed tomography (CT). Methods A cohort of 120 children aged 1-17 years underwent emergency CT, including the chest, after highenergy trauma. Axial 5-mm reconstructions were evaluated for lymph nodes at hilar and various mediastinal levels and the short-axis diameters were measured.

Results At least one lymph node was found in 115 (96\%) children, with subcarinal (69\%), lower paratracheal (64\%) and hilar $(60 \%)$ nodes being most common. Up to 10 years of age most lymph nodes were smaller than or equal to $7 \mathrm{~mm}$. In older children lymph nodes measuring up to 10 $\mathrm{mm}$-short-axis diameter were found. Lymph nodes were rare along the mammary vessels, at lower oesophageal and at prevascular and posterior mediastinal levels in children. Conclusion Mediastinal and hilar lymph nodes are more common than previously thought, probably because of increased detection by modern multi-detector CT. Lymph node location and age have to be taken into account when evaluating lymph nodes in the paediatric chest.

Key Points

- The size and location of normal lymph nodes is of great diagnostic importance

- There is little relevant published information related to pediatric chest computed tomography
\end{abstract}

P. A. de Jong $(\varangle) \cdot$ R.-J. A. Nievelstein

Department of Radiology, University Medical Center

Utrecht and Wilhelmina Children's Hospital,

HP E.01.132, Heidelberglaan 100,

3584 CX Utrecht, The Netherlands

e-mail: pimdejong@gmail.com
- We provide normative data concerning mediastinal and hilar lymph nodes in children

- These normal values can help identify abnormal lymph nodes in children

Keywords Lymphadenopathy · Pediatric · Chest computed tomography $\cdot$ Mediastinum $\cdot$ Normal measurement

\section{Introduction}

Lymphadenopathy can be an important sign of malignant or infectious disease in children. For the diagnosis of abnormally enlarged lymph nodes on chest CT, normal short-axis diameters for various lymph node locations for various ages should be known. For that purpose almost two decades ago Miller et al. studied 213 children aged 1 week to 20 years who did not have mediastinal abnormalities on CT [1]. They did not see any lymph nodes in the mediastinum on the CTs of these children. Around the same time Delacourt et al. studied 10 children with a primary bone tumour, congenital heart disease or mediastinal lipoma and no bronchopulmonary infection in the preceding 6 weeks [2]. They detected lymph nodes only at the right pretracheal and hilar levels and defined abnormal as sizes $\geq 5 \mathrm{~mm}$ and $\geq 4 \mathrm{~mm}$ in children below 4 years of age and $\geq 7 \mathrm{~mm}$ and $\geq 6 \mathrm{~mm}$ in children above 8 years of age, respectively. However, advances in CT technology may make more normal lymph nodes visible and therefore data need to be updated based on multidetector CT technology. The need for normative data has been acknowledged [3], but studies are scarce, because it is ethically challenging to expose healthy paediatric subjects to $\mathrm{CT}$ radiation to obtain normal measurements for mediastinal and hilar lymph nodes. We argued that 
children who undergo emergency CT because of highenergy trauma can be seen as a sample of the normal paediatric population and that those CTs can provide an insight into the normal dimensions of lymph nodes at various levels in the chest.

Therefore, we aimed to study lymph nodes on multidetector chest $\mathrm{CT}$ in children with high-energy trauma to provide normal measurements for lymph nodes.

\section{Materials and methods}

\section{Subjects}

All children up to 17 years of age who underwent multidetector CT of the chest after high-energy trauma in the emergency setting in a single centre between April 2006 and March 2011 were included. One patient was excluded because of a large mediastinal haematoma that compromised the evaluation of mediastinal lymph nodes. Ethical approval and informed consent are not required for this type of research in our country.

\section{CT}

The CT examinations were obtained with $16 \times 0.75 \mathrm{~mm}$ collimation (Mx8000 IDT or Brilliance 16P), $64 \times 0.625 \mathrm{~mm}$ collimation (Brilliance 64 ) or $128 \times 0.625 \mathrm{~mm}$ collimation (Brilliance iCT, all from Philips Medical Systems, Cleveland, $\mathrm{OH}, \mathrm{USA}$ ). Exposure settings were adjusted to patient size and ranged from 40 to $360 \mathrm{mAs}$ and from 80 to $140 \mathrm{kV}$. Axial images with slice-thickness of $5.0 \mathrm{~mm}$ were reconstructed from lung apices to lung bases. All patients received intravenous contrast medium, except 7 (6\%) who underwent $\mathrm{CT}$ to evaluate fractures.

\section{CT measurements}

One observer with 9 years' experience in paediatric and chest imaging evaluated all CT examinations and measured the short axis of the lymph nodes using an electronic calliper on a routine diagnostic screen. For each of the following locations the short axis of the largest lymph node was measured.

- Upper paratracheal between the top of the aortic arch and lung apex

- Prevascular and posterior mediastinal

- Lower paratracheal between top of the aortic arch and carina

- Aorto-pulmonary

- Subcarinal

- Hilar
- Along the mammary vessels

- Para-oesophageal below the level of the carina

Data analysis

The size of the lymph nodes was analysed per location and age group by using the Spearman correlation coefficient. SPSS 15.0 (Inc. Chicago, IL, USA) was used for data analysis. Significance level was set at $p<0.05$.

\section{Results}

At least one lymph node was measured in $115(96 \%)$ children. Lymph nodes were common subcarinal (69\%), lower paratracheal (64\%), hilar (60\%), upper paratracheal $(46 \%)$ and aorto-pulmonary (26\%). We found a prevascular lymph node in $1(0.8 \%)$, lower para-oesophageal lymph nodes in $7(6 \%)$ and mammary in none $(0 \%)$ of the patients. The size of the lymph nodes increased with age (Fig. 1, Table 1); Spearman correlation was $0.34(p=0.01), 0.42$ $(p<0.001), 0.43 \quad(p=0.02), 0.47 \quad(p<0.001)$ and 0.57 $(p<0.001)$ for upper paratracheal, lower paratracheal, aorto-pulmonary, subcarinal and hilar levels, respectively. Below the age of 10 years maximum short-axis diameter was $7 \mathrm{~mm}$ in the mediastinum and $6 \mathrm{~mm}$ at the hilar levels. From the age of 10 years these maximum diameters were $10 \mathrm{~mm}$ and $9 \mathrm{~mm}$, respectively.

\section{Discussion}

Lymph nodes are common on multi-detector CT of the chest in children. We provide prevalence and short-axis diameters per location and age for children aged 1-17 years, which updates earlier work with previous generations of CT systems. This update is important as lymph nodes are visible at more mediastinal stations than previously thought $[1,2]$. Caution is needed when identifying abnormal lymph nodes at high paratracheal and aorto-pulmonary levels, as we found, contrary to the older studies, lymph nodes at these levels in a substantial number of children. Also, nodes can be seen at pre-vascular and at lower para-oesophageal levels on rare occasions. Maximum short-axis diameter of 'normal' lymph nodes increases with age and the cut-off value to define a lymph node as enlarged is lower in young children confirming previous studies [2]. For adolescents it is reasonable to use, similar to most adult studies, a $10-\mathrm{mm}$ short-axis diameter as the upper limit of normal for hilar and mediastinal lymph nodes, although one has to realise that at most mediastinal levels it was rare to find lymph nodes larger than $7 \mathrm{~mm}$. For children up to 10 years of age 
Fig. 1 Size of lymph nodes on multi-detector $\mathrm{CT}$ in 120 children at various mediastinal and hilar levels. The $x$-axis represents age in years and the $y$-axis represents the short-axis diameter in millimetres
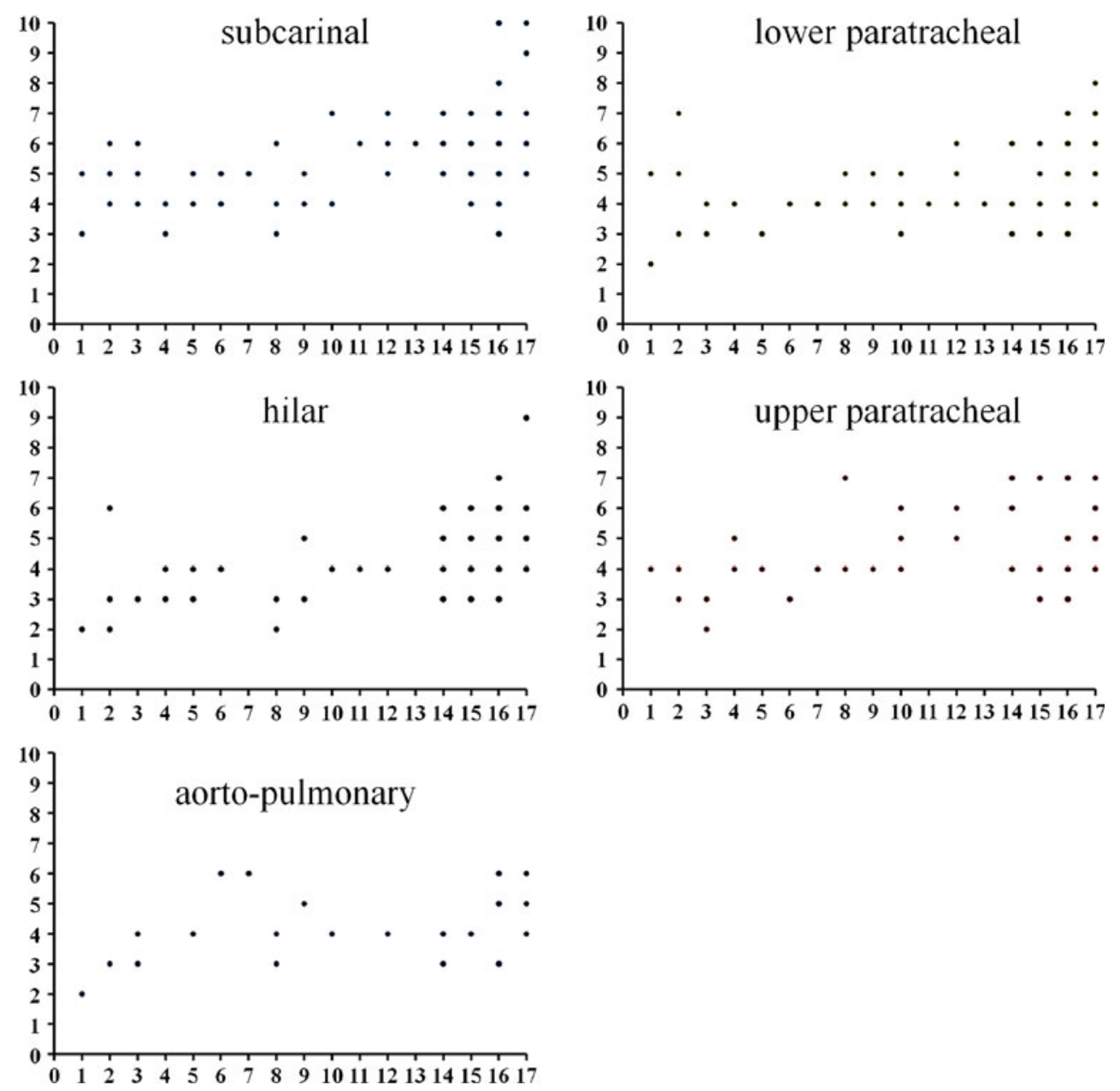

Table 1 Prevalence and size of lymph nodes in the chest on multi-detector CT in 120 children

\begin{tabular}{|c|c|c|c|c|c|c|c|c|c|c|c|c|}
\hline \multirow{2}{*}{$\begin{array}{l}\text { Age } \\
\text { Years }\end{array}$} & \multirow{2}{*}{$\begin{array}{l}\text { Subjects } \\
n\end{array}$} & \multirow{2}{*}{$\begin{array}{l}\text { Any lymph node } \\
n(\%)\end{array}$} & \multicolumn{2}{|c|}{ High paratracheal } & \multicolumn{2}{|c|}{ Low paratracheal } & \multicolumn{2}{|c|}{ Aorto-pulmonary } & \multicolumn{2}{|c|}{ Subcarinal } & \multicolumn{2}{|l|}{ Hilar } \\
\hline & & & $n(\%)$ & Size & $n(\%)$ & Size & $n(\%)$ & Size & $n(\%)$ & Size & $n(\%)$ & Size \\
\hline 1 & 3 & $3(100)$ & $1(33)$ & 4 & $2(67)$ & 5 & $1(33)$ & 2 & $2(67)$ & 5 & $1(33)$ & 2 \\
\hline 2 & 7 & $7(100)$ & $4(57)$ & 4 & $5(71)$ & 7 & $1(14)$ & 3 & $5(71)$ & 6 & $5(71)$ & 6 \\
\hline 3 & 4 & $4(100)$ & $2(50)$ & 3 & $3(75)$ & 4 & $3(75)$ & 4 & $3(75)$ & 6 & $1(25)$ & 3 \\
\hline 4 & 3 & $3(100)$ & $2(67)$ & 5 & $2(67)$ & 4 & $0(0)$ & & $3(100)$ & 4 & $2(67)$ & 4 \\
\hline 5 & 4 & $3(75)$ & $1(25)$ & 4 & $1(25)$ & 3 & $1(25)$ & 4 & $2(50)$ & 5 & $2(50)$ & 4 \\
\hline 6 & 3 & $3(100)$ & $1(33)$ & 3 & $2(67)$ & 4 & $1(33)$ & 6 & $2(67)$ & 5 & $2(67)$ & 4 \\
\hline 7 & 2 & $2(100)$ & $1(50)$ & 4 & $2(100)$ & 4 & $1(50)$ & 6 & $2(100)$ & 5 & $0(0)$ & \\
\hline 8 & 5 & $5(100)$ & $3(60)$ & 7 & $4(80)$ & 5 & $2(40)$ & 4 & $3(60)$ & 6 & $3(60)$ & 3 \\
\hline 9 & 2 & $2(100)$ & $1(50)$ & 4 & $2(100)$ & 5 & $1(50)$ & 5 & $2(100)$ & 5 & $2(100)$ & 5 \\
\hline 10 & 7 & $7(100)$ & $3(43)$ & 6 & $5(71)$ & 5 & $2(29)$ & 4 & $4(57)$ & 7 & $3(43)$ & 4 \\
\hline 11 & 3 & $1(33)$ & $0(0)$ & & $1(33)$ & 4 & $0(0)$ & & $1(33)$ & 6 & $1(33)$ & 4 \\
\hline 12 & 5 & $5(100)$ & $2(40)$ & 6 & $4(80)$ & 6 & $1(20)$ & 4 & $3(60)$ & 7 & $2(40)$ & 4 \\
\hline 13 & 1 & $1(100)$ & $0(0)$ & & $1(100)$ & 4 & $0(0)$ & & $1(100)$ & 6 & $0(0)$ & \\
\hline 14 & 7 & $7(100)$ & $3(43)$ & 7 & $5(71)$ & 6 & $2(29)$ & 4 & $5(71)$ & 7 & $5(71)$ & 6 \\
\hline 15 & 13 & $13(100)$ & $8(62)$ & 7 & $9(69)$ & 6 & $2(15)$ & 4 & $7(54)$ & 7 & $11(85)$ & 6 \\
\hline 16 & 26 & $25(96)$ & $10(38)$ & 7 & $15(58)$ & 7 & $8(31)$ & 6 & $19(73)$ & 10 & $16(62)$ & 7 \\
\hline 17 & 25 & $24(96)$ & $13(52)$ & 7 & $14(56)$ & 8 & $5(20)$ & 6 & $16(64)$ & 10 & $16(64)$ & 9 \\
\hline
\end{tabular}

Size is given in millimetres for the largest lymph node at that level measured by its short axis 
$7 \mathrm{~mm}$ is a reasonable upper limit of normal for mediastinal and hilar lymph nodes.

Our study has some limitations. We did not prospectively carry out $\mathrm{CT}$ in a normal healthy population, nevertheless, we believe our strategy is a reasonable alternative given the ethical constraints with radiation exposure in this age group. Also, we did not have information on previous illness or recent infections, which can be a cause of the visibility of these lymph nodes, but in our opinion there are two reasons why this does not invalidate our results. First, lack of such knowledge represents routine practice and a (recent) common cold is not a reason to postpone a chest CT. Second, the high prevalence of identifiable lymph nodes excludes the possibility that previous illness or recent infections can explain the lymph nodes in most of our subjects. Another limitation may be that we used our routine $5-\mathrm{mm}$ axial reconstruction and thinner slices may lead to the detection of even more lymph nodes. However, we already found nodes in most of the children and especially the larger nodes (upper limit of normal) would not be missed on the 5-mm slices. Finally, we included CTs from the emergency setting, but we find it unreasonable to assume that lymph nodes grow within minutes to hours after high-energy trauma and our subjects underwent imaging in the emergency room setting.
In conclusion, when evaluating lymph nodes in the paediatric chest on multi-detector CT one has to realise that lymph nodes are common at many mediastinal and hilar locations and that the size of normal lymph nodes is, not surprisingly, smaller in young children than that quoted for adolescents and adults. This may aid the diagnosis of lymphadenopathy in childhood.

Open Access This article is distributed under the terms of the Creative Commons Attribution Noncommercial License which permits any noncommercial use, distribution, and reproduction in any medium, provided the original author(s) and source are credited.

\section{References}

1. Miller FH, Fitzgerald SW, Donaldson JS (1993) CT of the azygoesophageal recess in infants and children. RadioGraphics 13:623-634

2. Delacourt C, Mani TM, Bonnerot V, de Blic J, Sayeg N, Lallemand D, Scheinmann P (1993) Computed tomography with normal chest radiograph in tuberculous infection. Arch Dis Child 69:430-432

3. Andronikou S (2002) Pathological correlation of CT-detected mediastinal lymphadenopathy in children: the lack of size threshold criteria for abnormality. Pediatr Radiol 32:912 